\title{
Detection Algorithms Based on Chip-Level Processing for DS/CDMA Code Acquisition in Fast Fading Channels *
}

\author{
Seokho Yoon ${ }^{1}$, Jee-Hyong Lee ${ }^{1}$, and Sun Yong Kim ${ }^{2}$ \\ 1 School of Information and Communication Engineering, Sungkyunkwan University, \\ 300 Chunchun-dong, Jangan-gu, Suwon, Kyunggi-do, 440-746, Korea \\ \{syoon, jhlee@ece.skku.ac.kr\} \\ 2 Department of Electronics Engineering, Konkuk University, \\ 1 Hwayang-dong, Gwangjin-gu, Seoul 143-701, Korea \\ kimsy@konkuk.ac.kr
}

\begin{abstract}
In this paper, we propose various novel detection algorithms based on chip-level processing for direct sequence code-division multiple access (DS/CDMA) pseudo noise (PN) code acquisition in fast fading channels, wherein the fading process changes rapidly within the accumulation interval of the correlation samples between the locally generated and received PN codes. By applying the maximum-likelihood (ML) and locally optimal (LO) detection criteria to the correlation samples obtained on a chip-by-chip basis, both optimal and LO detection algorithms are derived. Both of these algorithms are found to include the conventional algorithm as a special case. Simpler suboptimal algorithms are also derived. Finally, numerical results show that the proposed algorithms can offer a substantial improvement over the conventional algorithm in fast fading channels.
\end{abstract}

\section{Introduction}

In direct sequence code-division multiple access (DS/CDMA) systems, rapid code acquisition is crucial, because data demodulation is possible only after code acquisition is performed. The basic unit in an acquisition system is a detector whose task is to identify, with a high degree reliability, the presence or not of alignment between the locally generated and received pseudo noise $(\mathrm{PN})$ codes. The conventional detector which has been employed for code acquisition incorporates a detection algorithm based on the accumulation of the correlation samples between the locally generated and received PN codes. The accumulation of these samples is performed over an $N$ (usually, $N \gg 1$ ) chips interval called the dwell time. Recently, with the allocation of higher frequency bands

\footnotetext{
* This work was supported by grant No. R01-2004-000-10690-0 from the Basic Research Program of the Korea Science \& Engineering Foundation. Dr. S.Y. Kim is the corresponding author.
} 
for mobile communications and/or the increasing relative velocity of user terminals with respect to either a base station or a satellite, some mobile channels (e.g., CDMA based mobile satellite channels) exhibit very fast fading such that the fading process may exhibit rapid changes within the dwell time [1]. In such a scenario, the fading fluctuations among the correlation samples become very significant and, consequently, the effective accumulation of the correlation samples cannot be achieved even when the locally generated and received PN codes are synchronized. Needless to say, this seriously deteriorates the performance of the acquisition systems. In [2] and [3], it was shown that the performance of acquisition systems employing the conventional detector in such a fast fading scenario can be improved by using a parallel search strategy and antenna arrays, as compared with that obtained using a serial search strategy and a single antenna, respectively. However, the performance is very sensitive to the fading rate and degrades substantially as the fading becomes faster, as a result of the conventional detector being employed. In this paper, novel detection algorithms which alleviate the fast fading effect are proposed for code acquisition. In the proposed algorithms, the correlation samples are efficiently processed at the chip level before their accumulation so that the fading fluctuations among the correlation samples can be alleviated and, consequently, the effective accumulation of the correlation samples over the dwell time can be achieved.

\section{Observation Model}

The typical structure of the PN code acquisition system is shown in Fig. 1. In the correlator, the received signal $r(t)$ is first down converted to baseband and then is correlated with the locally generated PN code. The complex baseband equivalent signal $r_{l}(t)$ of $r(t)$ may be expressed as

$$
r_{l}(t)=\sqrt{P} \alpha(t) e^{j 2 \pi f_{0} t} d\left(t-\tau T_{c}\right) c\left(t-\tau T_{c}\right)+w(t) .
$$

In (1), $P$ is the transmitted signal power; $\alpha(t)$ is the (complex-valued) fading process; $f_{o}$ is the frequency offset between the transmitter and receiver; $d(t)$ is the data waveform; $T_{c}$ is the chip duration; $c(t)$ is the $\mathrm{PN}$ code waveform with a period of $L$ chips; $\tau$ is the code phase normalized to $T_{c}$; and $w(t)$ is a zeromean complex additive white Gaussian noise (AWGN) with one-sided power

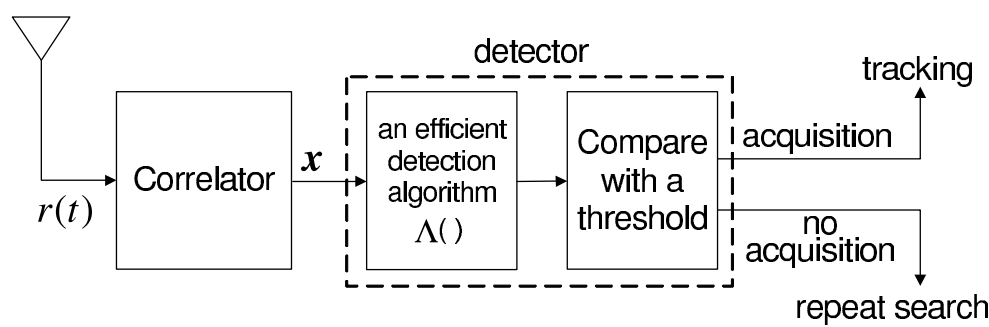

Fig. 1. Structure of PN code acquisition systems 
spectral density $N_{0}$. The noise process $w(t)$ represents noise plus multiple access interference and is independent of $\alpha(t)$. In this paper, the fading process $\alpha(t)$ is assumed to have a Rayleigh fading envelope and a uniformly distributed phase, and to be wide-sense stationary. Then, $\alpha(t)$ can be modeled as a zero-mean complex Gaussian random process with the autocorrelation function given as $\phi(\Delta t)=E\left\{\alpha(t) \alpha^{*}(t+\Delta t)\right\}$, where $E\{\cdot\}$ and $*$ denote the statistical expectation and the complex conjugate, respectively, and $\phi(0)=1$ due to normalization [4]. It is also assumed that there is a preamble for acquisition, so that no data modulation is present during acquisition, i.e., $d(t)=1$. The baseband signal $r_{l}(t)$ is now correlated with the locally generated PN code and then sampled on a chip-by-chip basis during the dwell time of $N$ chips. For simplicity, we assume that the system is chip synchronous and that the fading process $\alpha(t)$ is constant over one chip duration. Let hypotheses $K$ and $H$ correspond to in-phase and outof-phase alignments, respectively, between the locally generated and received PN codes. Then, the $k$ th correlation sample $x_{k}$, for $k=1,2, \cdots, N$, is given by

$$
\begin{aligned}
x_{k} & =\frac{1}{T_{c}} \int_{(k-1) T_{c}}^{k T_{c}} r_{l}(t) c\left(t-\hat{\tau} T_{c}\right) d t \\
& = \begin{cases}\sqrt{P} \alpha_{k} e^{j \pi \epsilon(2 k-1) \frac{\sin (\pi \epsilon)}{\pi \epsilon}+w_{k},} & \text { under } K \\
w_{k}, & \text { under } H,\end{cases}
\end{aligned}
$$

where $\hat{\tau}$ is the code phase (normalized to the chip duration $T_{c}$ ) of the locally generated $\mathrm{PN}$ code, $\epsilon$ is the frequency offset normalized to the chip rate $T_{c}^{-1}$, $\left\{\alpha_{k}\right\}_{k=1}^{N}$ are zero-mean complex Gaussian random variables with autocorrelation function $\phi\left(|m-n| T_{c}\right)=E\left\{\alpha_{m} \alpha_{n}^{*}\right\}$, and $\left\{w_{k}\right\}_{k=1}^{N}$ are zero-mean independent and identically distributed (i.i.d.) complex Gaussian random variables with variance $\sigma_{w}^{2}=N_{0} T_{c}^{-1}$. From (2), it is easy to see that $\left\{x_{k}\right\}_{k=1}^{N}$ are jointly complex Gaussian distributed and, thus, the pdf, $f_{\boldsymbol{x}}(\boldsymbol{x})$, of the correlation sample vector, $\boldsymbol{x}=\left(x_{1}, x_{2}, \cdots, x_{N}\right)^{\mathcal{T}}$, with $(\cdot)^{\mathcal{T}}$ denoting the transpose, is given by

$$
f_{\boldsymbol{x}}(\boldsymbol{x})= \begin{cases}\frac{1}{\pi^{N} \operatorname{det}\left(\boldsymbol{\Gamma}_{K}\right)} \exp \left(-\boldsymbol{x}^{\mathcal{H}} \boldsymbol{\Gamma}_{K}^{-1} \boldsymbol{x}\right), & \text { under } K \\ \frac{1}{\pi^{N} \operatorname{det}\left(\boldsymbol{\Gamma}_{H}\right)} \exp \left(-\boldsymbol{x}^{\mathcal{H}} \boldsymbol{\Gamma}_{H}^{-1} \boldsymbol{x}\right), & \text { under } H\end{cases}
$$

where $\operatorname{det}(\cdot)$ and $(\cdot)^{\mathcal{H}}$ denote the determinant of a matrix and the Hermitian transpose, respectively. The elements located at row $m$ and column $n$ of the covariance matrices $\boldsymbol{\Gamma}_{K}=E\left\{\boldsymbol{x} \boldsymbol{x}^{\mathcal{H}} \mid K\right\}$ and $\boldsymbol{\Gamma}_{H}=E\left\{\boldsymbol{x} \boldsymbol{x}^{\mathcal{H}} \mid H\right\}$ are given by $P \phi\left(|m-n| T_{c}\right) e^{j 2 \pi \epsilon(m-n)} \frac{\sin ^{2}(\pi \epsilon)}{\pi^{2} \epsilon^{2}}+\sigma_{w}^{2} \delta(m-n)$ and $\sigma_{w}^{2} \delta(m-n)$, respectively, where $\delta(\cdot)$ denotes the Kronecker delta function. Finally, a detection algorithm $\Lambda(\cdot)$ is performed with the correlation sample vector $\boldsymbol{x}$, and then its outcome is compared with a threshold. The conventional detection algorithm, denoted by $\Lambda_{\mathrm{C}}(\boldsymbol{x})$, is given by $\Lambda_{\mathrm{C}}(\boldsymbol{x})=\boldsymbol{x}^{\mathcal{H}} \mathbf{1}_{N \times N} \boldsymbol{x}=\left|\sum_{k=1}^{N} x_{k}\right|^{2}$, where $\mathbf{1}_{N \times N}$ denotes an all-one matrix with size $N \times N$. It should be noted that in $\Lambda_{\mathrm{C}}(\boldsymbol{x})$ no processing is performed on the correlation samples before accumulation. 


\section{Optimal and Locally Optimal Detection Algorithms}

\subsection{Chip-Level Optimal (CLO) Detection Algorithm}

A chip-level optimal (CLO) detection algorithm is derived using the maximum likelihood (ML) detection criterion. Using (3), the log likelihood ratio test can be written as follows: $\ln \left(\frac{f_{\boldsymbol{x}}(\boldsymbol{x} \mid K)}{f_{\boldsymbol{x}}(\boldsymbol{x} \mid H)}\right)=\ln \left(\frac{\operatorname{det}\left(\boldsymbol{\Gamma}_{H}\right)}{\operatorname{det}\left(\boldsymbol{\Gamma}_{K}\right)}\right)+\boldsymbol{x}^{\mathcal{H}}\left(\boldsymbol{\Gamma}_{H}^{-1}-\boldsymbol{\Gamma}_{K}^{-1}\right) \boldsymbol{x}$, in which the first term, $\ln \left(\frac{\operatorname{det}\left(\boldsymbol{\Gamma}_{H}\right)}{\operatorname{det}\left(\boldsymbol{\Gamma}_{K}\right)}\right)$, does not depend on $\boldsymbol{x}$. Thus, the CLO detection algorithm, denoted by $\Lambda_{\mathrm{CLO}}(\boldsymbol{x})$, may be obtained as

$$
\Lambda_{\mathrm{CLO}}(\boldsymbol{x})=\boldsymbol{x}^{\mathcal{H}}\left(\boldsymbol{\Gamma}_{H}^{-1}-\boldsymbol{\Gamma}_{K}^{-1}\right) \boldsymbol{x}=\boldsymbol{x}^{\mathcal{H}}\left(\sigma_{w}^{-2} \mathbf{I}_{N}-\left(\sigma_{w}^{2} \mathbf{I}_{N}+\gamma^{2} \mathbf{R}_{\boldsymbol{s}}\right)^{-1}\right) \boldsymbol{x},
$$

where $\mathbf{I}_{N}$ denotes the identity matrix of size $N, \gamma=\sqrt{P} \frac{\sin (\pi \epsilon)}{\pi \epsilon}$, and $\mathbf{R}_{\boldsymbol{s}}=$ $E\left\{\boldsymbol{s s}^{\mathcal{H}}\right\}$ with $\boldsymbol{s}=\left(\alpha_{1} e^{j \pi \epsilon}, \alpha_{2} e^{j 3 \pi \epsilon}, \cdots, \alpha_{N} e^{j(2 N-1) \pi \epsilon}\right)^{\mathcal{T}}$. Under the assumption that the fading remains constant during the dwell time of $N$ chips and that there is no frequency offset, $\mathbf{R}_{\boldsymbol{s}}$ can be simplified to $\mathbf{1}_{N \times N}$. Then, applying the Sherman-Morrison-Woodbury formula [5] to the term $\left(\sigma_{w}^{2} \mathbf{I}_{N}+\gamma^{2} \mathbf{R}_{\boldsymbol{s}}\right)^{-1}$ in (4) gives $\left(\sigma_{w}^{2} \mathbf{I}_{N}+\gamma^{2} \mathbf{1}_{N \times N}\right)^{-1}=\sigma_{w}^{-2} \mathbf{I}_{N}-\mathrm{C} \mathbf{1}_{N \times N}$, where $\mathrm{C}=\frac{\gamma^{2}}{\sigma_{w}^{4}+N \gamma^{2} \sigma_{w}^{2}}$. Substituting this result into (4), we find that $\Lambda_{\mathrm{CLO}}(\boldsymbol{x})$ can be expressed as $\boldsymbol{x}^{\mathcal{H}} \mathbf{1}_{N \times N} \boldsymbol{x}=\left|\sum_{k=1}^{N} x_{k}\right|^{2}$, which is the conventional detection algorithm, $\Lambda_{\mathrm{C}}(\boldsymbol{x})$, mentioned in the previous section. This means that $\Lambda_{\mathrm{CLO}}(\boldsymbol{x})$ includes $\Lambda_{\mathrm{C}}(\boldsymbol{x})$ as a special case, wherein the fading remains constant during the dwell time and there is no frequency offset.

\subsection{Chip-Level Locally Optimal (CLLO) Detection Algorithm}

As shown in (4), $\Lambda_{\mathrm{CLO}}(\boldsymbol{x})$ requires the inversion of the $N \times N$ matrix. To derive an algorithm which is simple compared with $\Lambda_{\mathrm{CLO}}(\boldsymbol{x})$, we use the locally optimal (LO) detection criterion, which leads to the LO detection algorithm in signal detection theory. The LO detection algorithm is usually much easier to implement than the optimal detection algorithms, and yields the maximum outcome value when the signal-to-noise ratio (SNR) approaches zero [6]. From the generalized Neyman-Pearson's fundamental lemma [6] and (3), we can obtain the chip-level locally optimal (CLLO) detection algorithm as

$$
\Lambda_{\mathrm{CLLO}}(\boldsymbol{x})=\left.\frac{1}{f_{\boldsymbol{x}}(\boldsymbol{x} \mid H)} \frac{d^{\nu} f_{\boldsymbol{x}}(\boldsymbol{x} \mid K)}{d \mu^{\nu}}\right|_{\mu=0}=\boldsymbol{x}^{\mathcal{H}} \mathbf{R}_{\boldsymbol{s}} \boldsymbol{x},
$$

where $\mu$ is a signal strength parameter (in this paper, we set $\mu=\gamma=\sqrt{P} \frac{\sin (\pi \epsilon)}{\pi \epsilon}$ ) and $\nu$ is the order of the first nonzero derivative of $f_{\boldsymbol{x}}(\boldsymbol{x} \mid K)$ at $\mu=0$. It is noteworthy that, in contrast to $\Lambda_{\mathrm{CLO}}(\boldsymbol{x}), \Lambda_{\mathrm{CLLO}}(\boldsymbol{x})$ does not require matrix inversion. When the fading remains constant during the dwell time of $N$ chips and there is no frequency offset, $\mathbf{R}_{\boldsymbol{s}}$ can be simplified to $\mathbf{1}_{N \times N}$ and, thus, $\Lambda_{\mathrm{CLLO}}(\boldsymbol{x})$ becomes $\Lambda_{\mathrm{C}}(\boldsymbol{x})$, from which we find that, as well as $\Lambda_{\mathrm{CLO}}(\boldsymbol{x}), \Lambda_{\mathrm{CLLO}}(\boldsymbol{x})$ also 
includes $\Lambda_{\mathrm{C}}(\boldsymbol{x})$ as a special case. It should be noted that $\Lambda_{\mathrm{CLO}}(\boldsymbol{x})$ and $\Lambda_{\mathrm{CLLO}}(\boldsymbol{x})$ need only the statistical description of the fading process, and not the actual realizations of the fading process, in compensating for the fast fading effect before combining the correlation samples. Such a requirement, however, may limit their implementation. Thus, in the next section, suboptimal detection algorithms are discussed, which obviate the need for any information on the fading statistics (and the frequency offset).

\section{Suboptimal Detection Algorithms}

\subsection{Chip-Level Noncoherent (CLN) Detection Algorithm}

From (4) and (5), we can observe that $\Lambda_{\mathrm{CLO}}(\boldsymbol{x})$ and $\Lambda_{\mathrm{CLLO}}(\boldsymbol{x})$ form a weighted sum of $\left\{x_{k}^{*} x_{l}\right\}_{k, l=1}^{N}$ through $\left(\boldsymbol{\Gamma}_{H}^{-1}-\boldsymbol{\Gamma}_{K}^{-1}\right)$ and $\mathbf{R}_{\boldsymbol{s}}$, respectively. $\Lambda_{\mathrm{CLLO}}(\boldsymbol{x})$, for example, can be rewritten as

$$
\Lambda_{\mathrm{CLLO}}(\boldsymbol{x})=\sum_{k=1}^{N}\left|x_{k}\right|^{2}+\sum_{k=1}^{N} \sum_{l=1, l \neq k}^{N} \phi\left(|k-l| T_{c}\right) e^{j 2 \pi \epsilon(k-l)} x_{k}^{*} x_{l} .
$$

In (6), it can be seen that $\Lambda_{\mathrm{CLLO}}(\boldsymbol{x})$ compensates for the combined effects of fading and frequency offset on $\left\{x_{k}^{*} x_{l}\right\}_{k, l=1}^{N}$ through the weighting factor $\phi\left(|k-l| T_{c}\right)$ $\cdot e^{j 2 \pi \epsilon(k-l)}$, and it is also observed that the components $\left\{\left|x_{k}\right|^{2}\right\}_{k=1}^{N}$ are added with equal weights regardless of fading time variation and frequency offset. From this observation, we propose to use the following algorithm as a suboptimal detection algorithm, which does not require any channel information:

$$
\Lambda_{\mathrm{CLN}}(\boldsymbol{x})=\sum_{k=1}^{N}\left|x_{k}\right|^{2}
$$

where CLN is an abbreviation for "chip-level noncoherent", which originates from the fact that $\left|x_{k}\right|^{2}$ can be considered as noncoherent processing performed at the chip level. In fact, $\Lambda_{\mathrm{CLN}}(\boldsymbol{x})$ becomes optimal and locally optimal for a fast fading channel such that all of the elements of $\boldsymbol{x}$ are uncorrelated: specifically, in such an environment, $\left(\boldsymbol{\Gamma}_{H}^{-1}-\boldsymbol{\Gamma}_{K}^{-1}\right)$ and $\mathbf{R}_{\boldsymbol{s}}$ can be simplified to $\frac{\gamma^{2} \sigma_{w}^{-2}}{\sigma_{w}^{2}+\gamma^{2}} \mathbf{I}_{N}$ and $\mathbf{I}_{N}$, respectively, and thus $\Lambda_{\mathrm{CLO}}(\boldsymbol{x})$ and $\Lambda_{\mathrm{CLLO}}(\boldsymbol{x})$ become $\frac{\gamma^{2} \sigma_{w}^{-2}}{\sigma_{w}^{2}+\gamma^{2}} \boldsymbol{x}^{\mathcal{H}} \mathbf{I}_{N} \boldsymbol{x}$ and $\boldsymbol{x}^{\mathcal{H}} \mathbf{I}_{N} \boldsymbol{x}$, respectively, which are equivalent to $\Lambda_{\mathrm{CLN}}(\boldsymbol{x})$ given in (7).

\subsection{Chip-Level Differential (CLD) Detection Algorithm}

Now, it should be observed that the second term of (6) can be considered as the sum of the components differentially processed at the chip level, with weights that depend on channel information. Using (2), the signal components of $\left\{x_{k}^{*} x_{l}\right\}_{k, l=1, k \neq l}^{N}$ can be expressed as $\left\{\gamma^{2} \alpha_{k}^{*} \alpha_{l} e^{-j 2 \pi \epsilon(k-l)}\right\}_{k, l=1, k \neq l}^{N}$. If the phase 
fluctuation due to fading between two successive correlation samples is not very significant, the signal components $\left\{\gamma^{2} \alpha_{k}^{*} \alpha_{k+1} e^{j 2 \pi \epsilon}\right\}_{k=1}^{N-1}$ (corresponding to $\left.\left\{x_{k}^{*} x_{k+1}\right\}_{k=1}^{N-1}\right)$ from among $\left\{\gamma^{2} \alpha_{k}^{*} \alpha_{l} e^{-j 2 \pi \epsilon(k-l)}\right\}_{k, l=1, k \neq l}^{N}$ would be approximately phase aligned. Moreover, they are of equal average strength. As a result, just as was done in the case of $\left\{\left|x_{k}\right|^{2}\right\}_{k=1}^{N}$, the components $\left\{x_{k}^{*} x_{k+1}\right\}_{k=1}^{N-1}$ can be added with equal weights to form an algorithm: yet, it should be pointed

out that the signal component of $\sum_{k=1}^{N-1} x_{k}^{*} x_{k+1}$ is divided into real and imaginary parts, in contrast to that of $\sum_{k=1}^{N}\left|x_{k}\right|^{2}$. Hence, we take the envelope of $\sum_{k=1}^{N-1} x_{k}^{*} x_{k+1}$ to combine the divided signal parts, and thus obtain a suboptimal detection algorithm such that

$$
\Lambda_{\mathrm{CLD}}(\boldsymbol{x})=\left|\sum_{k=1}^{N-1} x_{k}^{*} x_{k+1}\right|
$$

where CLD is an abbreviation for "chip-level differential". $\Lambda_{\mathrm{CLD}}(\boldsymbol{x})$ is expected to be more sensitive to the fading rate than $\Lambda_{\mathrm{CLN}}(\boldsymbol{x})$, since the degree of phase coherence among the signal components of $\left\{x_{k}^{*} x_{k+1}\right\}_{k=1}^{N-1}$ depends on the fading rate.

\section{Simulation Results and Discussion}

We compare the detection performance of the conventional and proposed detection algorithms. In evaluating the performance, we consider the following parameters: the PN code of $L=1023$ chips, the dwell time length $N=256$ chips, and the false alarm probability $P_{F}=10^{-2}$. The SNR/chip is defined as $P T_{c} / N_{0}$. The autocorrelation function, $\phi(\Delta t)$, of the fading process is taken as $\rho^{\Delta t / T_{c}}[2],[3]$, where $0 \leq \rho \leq 1$ is the parameter that characterizes the fading rate, such that the smaller the value of $\rho$, the faster the fading.

Fig. 2 shows the detection probabilities of the conventional and proposed detection algorithms for $\rho=0.97$ and 0.95 in the absence of frequency offset. As expected, the performance of the conventional algorithm degrades substantially as $\rho$ becomes smaller, i.e., the fading becomes faster, whereas the performance of the proposed algorithms improves as the fading becomes faster; however, an opposite trend occurs at relatively low SNR/chip values. This can be explained as follows. As the fading becomes faster, the chip-level processed components become more uncorrelated, and thus the diversity gain obtained through the combining of the components increases, resulting in better detection performance. However, the increased fading rate enhances the phase misalignments among the differentially processed components and makes the noncoherent combining loss [4] for the noncoherently processed components more pronounced. Eventually, at low SNR/chip values, such effects become more significant than the diversity effect, resulting in worse detection performance. 


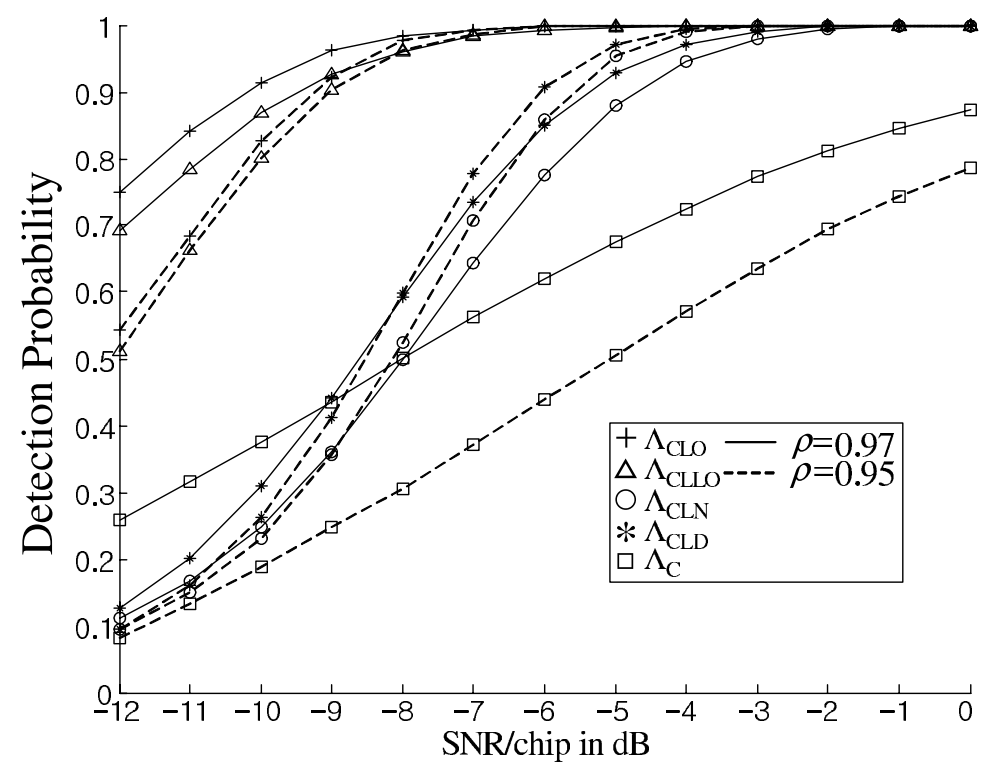

Fig. 2. Detection probability of the conventional and proposed detection algorithms for $\rho=0.97$ and 0.95 when $\epsilon=0$

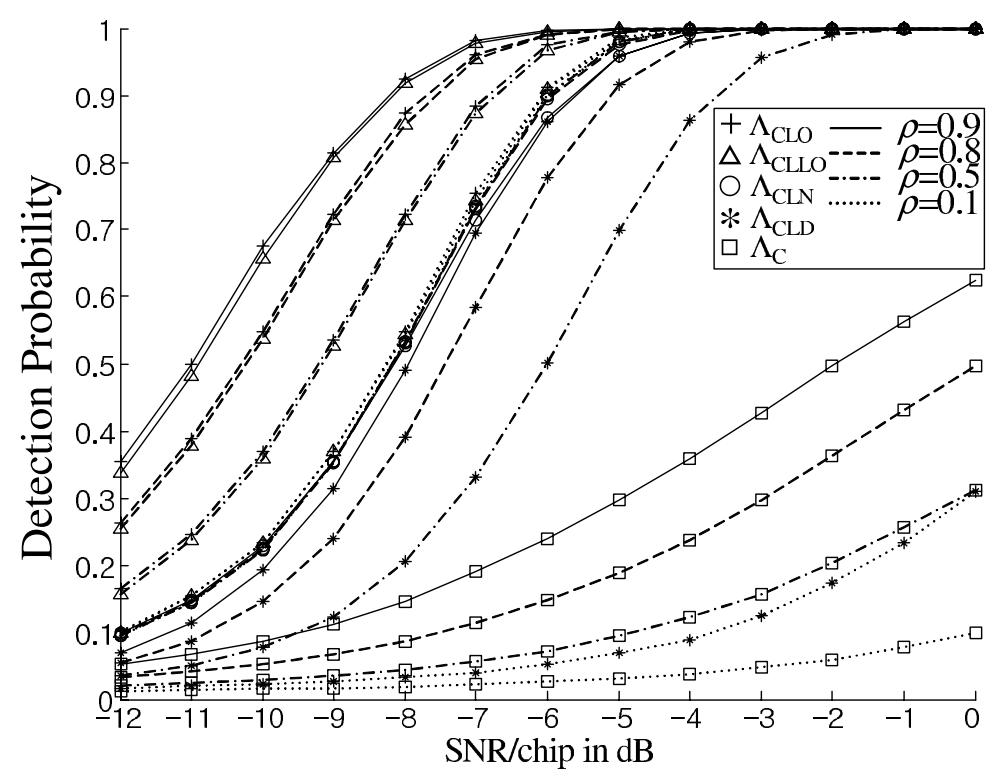

Fig. 3. Detection probability of the conventional and proposed detection algorithms for $\rho=0.9,0.8,0.5$, and 0.1 with $\epsilon=0.001$ 
Fig. 3 shows the detection probabilities of the conventional and proposed detection algorithms for $\rho=0.9,0.8,0.5$, and 0.1 with $\epsilon=0.001$. From this figure, we can observe that the performance of the conventional algorithm degrades severely due to fading and frequency offset. Unlike in Fig. 2, as the fading becomes faster, the performances of $\Lambda_{\mathrm{CLO}}(\boldsymbol{x}), \Lambda_{\mathrm{CLLO}}(\boldsymbol{x})$, and $\Lambda_{\mathrm{CLD}}(\boldsymbol{x})$ are observed to degrade for the whole range of SNR/chip values shown. This is due to the fact that, as the fading becomes faster, the extent of the phase misalignments among the differentially processed components increases and its effect becomes predominant over the diversity gain effect regardless of the SNR/chip value. On the other hand, the performance of $\Lambda_{\mathrm{CLN}}(\boldsymbol{x})$ follows the same trend as that shown in Fig. 2. Finally, the performance of $\Lambda_{\mathrm{CLN}}(\boldsymbol{x})$ is found to be quite robust to variations in the value of $\rho$ and to approach that of $\Lambda_{\mathrm{CLO}}(\boldsymbol{x})$ and $\Lambda_{\mathrm{CLLO}}(\boldsymbol{x})$ as the fading becomes faster, as stated in Subsection 4.1.

\section{Conclusion}

In this paper, various detection algorithms were proposed based on chip-level processing for DS/CDMA code acquisition in fast fading channels, wherein the fading process changes rapidly within the dwell time. First, we derived the joint pdf of the correlation samples obtained on a chip-by-chip basis during the dwell time. Based on this pdf and on the ML and LO detection criteria, chip-level optimal and chip-level LO detection algorithms were proposed, which require the statistics, but do not need the realization of the fading parameters. Both algorithms were found to include the conventional algorithm as a special case, wherein the fading process remains constant during the dwell time and there is no frequency offset. Two suboptimal detection algorithms were also derived. The proposed detection algorithms were shown to dramatically outperform the conventional detection algorithm as the fading becomes faster.

\section{References}

1. T.K. Sarkar, Z. Ji, K. Kim, A. Medouri, and M. Salazar-palma, "A survey of various propagation models for mobile communication," IEEE Anten. Propag. Mag., vol. 45, pp. 51-82, June 2003.

2. E.A. Sourour and S.C. Gupta, "Direct-sequence spread-spectrum parallel acquisition in a fading mobile channel," IEEE Trans. Comm., vol. 38, pp. 992-998, July 1990.

3. W.H. Ryu, M.K. Park, and S.K. Oh, "Code acquisition schemes using antenna arrays for DS-SS systems and their performance in spatially correlated fading channels," IEEE Trans. Comm., vol. 50, pp. 1337-1347, Aug. 2002.

4. J.G. Proakis, Digital Communications, NY: McGraw-Hill, 2001.

5. G.H. Golub and C.F. van Loan, Matrix Computations, MD: Johns Hopkins University Press, 1996.

6. S.A. Kassam, Signal Detection in Non-Gaussian Noise, NY: Springer-Verlag, 1987. 